\title{
Association between homocysteine and non-alcoholic fatty liver disease in Chinese adults: a cross-sectional study
}

Haijiang Dai ${ }^{1,2}$, Weijun Wang ${ }^{3}$, Xiaohong Tang ${ }^{1}$, Ruifang Chen ${ }^{1}$, Zhiheng Chen ${ }^{2}$, Yao Lu ${ }^{1 *}$ and Hong Yuan ${ }^{1 *}$

\begin{abstract}
Background: Non-alcoholic fatty liver disease (NAFLD) is the most common liver disease worldwide, and its prevalence is likely to rise even further. To help understand the pathogenesis and early prevention of progressive NAFLD, this large-scale study was designed to explore the potential association between homocysteine and the prevalence of NAFLD.

Methods: A total of 7203 subjects aged 18 years or older were enrolled in this cross-sectional study. The association of homocysteine with the prevalence of NAFLD, in the total sample and stratified by subgroups, was examined using multiple logistic regression analyses.

Results: Subjects in the higher quartiles of homocysteine had a higher prevalence of NAFLD. After multivariate adjustment, the odds ratio (OR) for NAFLD in the highest compared with the lowest quartile of homocysteine was 2.08 (95\% confidence interval [CI] 1.61, 2.67). Moreover, in the subgroup analyses, we found an effect modification by gender, body mass index (BMI) and smoking status on the association between homocysteine and the prevalence of NAFLD ( $P$ for interaction: 0.001, 0.002 and $<0.001$, respectively). A stronger association was observed in female, obese and non-smoking adults than in male, normal weight and smoking subjects.
\end{abstract}

Conclusion: Homocysteine was significantly associated with the prevalence of NAFLD, particularly in female, obese or non-smoking adults.

Keywords: Homocysteine, Non-alcoholic fatty liver disease, Gender, Body mass index, Interaction

\section{Background}

Non-alcoholic fatty liver disease (NAFLD), which encompasses a spectrum of conditions associated with lipid deposition in hepatocytes, is the most common liver disease. Worldwide, the overall prevalence of NAFLD diagnosed by imaging was $25.2 \%$ with the highest prevalence rates were reported from South America (30.5\%) and the Middle East (31.8\%) [1]. In China, NAFLD affects over a quarter of the population, and its prevalence is still increasing rapidly as a result of considerable changes in lifestyle and aging [2-4]. NAFLD is often associated with metabolic risk factors, such as obesity, type two diabetes, dyslipidemia, and insulin resistance.

\footnotetext{
*Correspondence: luyao0719@hotmail.com; yuanhong_xy3@163.com ${ }^{1}$ Center of Clinical Pharmacology, the Third Xiangya Hospital, Central South University, 138 Tong-Zi-Po Road, Changsha, Hunan Zip 410013, People's Republic of China

Full list of author information is available at the end of the article
}

In addition to hepatic complications, NAFLD is also associated with serious systemic consequences. NAFLD has been widely accepted to significantly increase the morbidity and mortality of cardiovascular diseases [5-7].

Homocysteine is a sulfhydryl-containing amino acid mainly produced and catabolized in the liver $[8,9]$. A growing body of evidence shows that homocysteine mediates cardiovascular problems by its adverse effects on cardiovascular endothelium and smooth muscle cells [10]. Moreover, homocysteine can alter intracellular lipid metabolism and may promote hepatic fat accumulation $[11,12]$. Thus, it is plausible that homocysteine could be an effective target for preventing the progression of NAFLD and its related cardiovascular complications. However, only a few studies have explored the association between homocysteine and the prevalence of NAFLD, and the answer remains controversial [13-15]. 
In addition, it is also important to confirm whether these associations are moderated by other closely related factors, such as gender and body mass index (BMI), as substantial differences exist with regard to the metabolism of homocysteine between males and females as well as among BMI groups $[10,16]$.

In this study, we performed a cross-sectional analysis to determine whether elevated homocysteine was associated with an increased prevalence of NAFLD in Chinese adults. Moreover, we also explored the possible effect modification by other related factors on the association between homocysteine and the prevalence of NAFLD.

\section{Methods}

\section{Study population}

The Health Management Center of Third Xiangya Hospital is one of China's largest examination centers, mainly servicing individuals from hundreds of institutions in Changsha. In this cross-sectional study, we consecutively recruited 13,916 individuals who underwent a health examination in the center from January 2014 to December 2014. The inclusion criteria were: 1) aged 18 years or older; 2) availability of abdominal ultrasonography examination; 3 ) and undergoing plasma homocysteine measurements. Questionnaires were answered by all of the enrolled subjects to collect information on medical history, alcohol consumption, cigarette smoking, etc. In order to remove the effect of alcohol on the fatty liver, we excluded subjects with excessive alcohol consumption $(n=1629)$ as well as those with incomplete information on alcohol consumption $(n=4451)$. As a result, 7836 subjects were included. Of these kept subjects, 565 subjects were diagnosed or selfreported to have viral hepatitis, schistosomiasis liver disease or other chronic liver diseases, 9 subjects were self-reported taking steatogenic medications, 12 subjects were self-reported taking vitamin B or folic acid, and 47 subjects had no available data on height or body weight; all of these patients were excluded from our study. Finally, 7203 subjects were screened and deemed eligible for the present study.

This study was approved by the Medical Ethics Committee of Third Xiangya Hospital. All of the experiments in this study were performed according to the guidelines from the Helsinki Declaration, and written informed consent was obtained from all participants.

\section{Data collection and measurements}

Information on age, gender, alcohol consumption, cigarette smoking, physical activity, education level, and medical history were obtained from standardized questionnaires through face-to-face interviews. Alcohol consumption was evaluated with questions regarding the types of alcoholic beverages, the frequency of alcohol consumption per week and the usual amount consumed per occasion. Subjects who reported alcohol consumption $\geq 140 \mathrm{~g} /$ week for men and $\geq 70 \mathrm{~g} /$ week for women were deemed to have excessive alcohol consumption [17]. Smoking was recorded as daily (at least one cigarette/day), occasional (less than one cigarette/day), former (having quit for at least 6 months), or never smoking. For our analyses, only two categories were considered: current smoking (daily and occasional smoking) and non-smoking (never and former smoking) [18]. Physical activity was defined as the frequency of physical activity during leisure time and was scored as inactive (1-2 times per week), moderate (3-5 times per week), or active ( $\geq 6$ times per week).

Standing height and body weight were measured without shoes or thick clothing, and BMI was calculated as body weight in kilograms divided by height in meters squared. Blood pressure (BP) was measured in the sitting position after a 10-min rest period using an appropriately sized cuff and a corrected mercury sphygmomanometer. Systolic BP and diastolic BP were each measured twice in this study, with a $30 \mathrm{~s}$ interval. If the two measurements differed by $>5 \mathrm{mmHg}$, BP was re-measured. Finally, the $\mathrm{BP}$ was calculated as the average of the three measurements. Hypertension was defined by the presence of any of the following: systolic BP $\geq 140 \mathrm{mmHg}$ and/or diastolic BP $\geq 90 \mathrm{mmHg}$, a history of hypertension, or current use of antihypertensive agents.

Venous blood sampling was performed after overnight fasting for $8-12 \mathrm{~h}$, and the blood glucose, alanine transaminase (ALT), total bilirubin (TBIL), albumin (ALB), platelet count (PLT), serum uric acid, triglyceride (TG), total cholesterol (TC), high-density lipoprotein cholesterol (HDL-C), high-sensitivity C-reactive protein (hs-CRP), serum creatinine and homocysteine levels were determined using standard laboratory methods. The laboratory methods were consistent throughout the study period. All blood samples were tested using an auto-analyzer (Hitachi 7600-110; Hitachi, Tokyo, Japan) at the central laboratory of Third Xiangya Hospital. Diabetes was diagnosed by fasting blood glucose $\geq 7.0 \mathrm{mmol} / \mathrm{L}$, a history of diabetes, or current use of hypoglycemic agents [19].

\section{Definition of non-alcoholic fatty liver disease}

Hepatic steatosis was diagnosed upon abdominal ultrasonography by experienced and trained radiologists who were blinded to the subjects' clinical diagnoses and biochemical tests. Positive abdominal ultrasound images included: diffusely increased liver near field ultrasound echo ('bright liver'), liver echo greater than kidney, vascular blurring and the gradual attenuation of far field ultrasound echo. Subjects with at least two of the abnormal findings listed above were diagnosed with hepatic steatosis $[17,20]$. Because all of the subjects with secondary causes for hepatic steatosis such as excessive alcohol consumption, viral hepatitis, or use of steatogenic 
medication were excluded from our study, NAFLD was defined by the presence of hepatic steatosis [20,21].

\section{Statistical analysis}

In order to derive a deeper understanding of the relationship between serum homocysteine levels and the prevalence of NAFLD, all of the study subjects were classified into four groups by their quartiles of homocysteine (Q1: $<5.1 \mu \mathrm{mol} / \mathrm{L}, \mathrm{Q} 2: 5.1$ to $<7.1 \mu \mathrm{mol} / \mathrm{L}, \mathrm{Q} 3$ : 7.1 to $<9.9 \mu \mathrm{mol} / \mathrm{L}, \mathrm{Q} 4: \geq 9.9 \mu \mathrm{mol} / \mathrm{L})$. Basic characteristics of the study subjects were presented as the mean \pm standard deviation (SD) or median (interquartile range) for continuous variables and as numbers with percentages for categorical variables. Variables that displayed a skewed distribution (age, ALT, TBIL, TG, hs-CRP and homocysteine) were log transformed to normal before analysis.

For continuous variables, one-way ANOVA with a post-hoc $t$-test with Bonferroni correction for multiple comparisons was used to compare differences between the quartiles of serum homocysteine. For categorical variables, differences between group frequencies were assessed with the Pearson $\chi^{2}$ test. The odds ratio (OR) with 95\% confidence intervals (CIs) were calculated using logistic regression to determine the risk of NAFLD for each quartile of homocysteine, with the lowest quartile regarded as the reference category. Moreover, age, gender, BMI (categorized as normal: $<24 \mathrm{~kg} / \mathrm{m}^{2}$, overweight: 24 to $<28 \mathrm{~kg} / \mathrm{m}^{2}$, and obesity: $\geq 28 \mathrm{~kg} / \mathrm{m}^{2}$, as based on the Working Group on Obesity in China guidelines [22]), current smoking, hypertension, and diabetes were evaluated to assess whether there was any significant interaction between these variables and the relationship between homocysteine levels and the prevalence of NAFLD.

All of the analyses were performed using IBM SPSS Statistics Ver. 22.0 (IBM Co., Armonk, NY, USA), and $P<0.05$ was considered to indicate statistical significance. Post-hoc Bonferroni correction was used for multiple comparisons.

\section{Results}

\section{Characteristics of the study subjects}

Of the 7203 subjects, 2370 (32.9\%) were diagnosed with NAFLD, and the mean homocysteine level was 7.0 (5.0, 9.9) $\mu \mathrm{mol} / \mathrm{L}$. The characteristics of the study population in total and according to homocysteine quartiles are displayed in Table 1. When compared with subjects in Q1 group, those with higher homocysteine levels were more likely to be older, male, and current smokers, and they tended to have increased prevalence of hypertension as well as higher levels of BMI, ALT, TBIL, ALB, serum uric acid, TG, hs-CRP and creatinine. In addition, the frequency of physical activity, prevalence of drinking and diabetes, and levels of education, PLT, TC, and HDL-C were also significantly different among the homocysteine quartiles.

\section{Association between homocysteine and non-alcoholic fatty liver disease}

As shown in Table 1, the prevalence of NAFLD progressively increased in the higher quartiles of homocysteine (19.8, 28.3, 37.7, and 46.6\%, respectively). Table 2 shows the multiple adjusted association between the quartiles of homocysteine and NAFLD. In the adjusted model 1, after adjustment for age, the OR for NAFLD in the highest compared to the lowest quartile of homocysteine was 3.10 (95\% CI 2.67, 3.59). After further adjustment for gender, BMI, current smoker, physical activity, education, drinking, hypertension, diabetes, uric acid, ALT, TBIL, ALB, PLT, TG, TC, HDL-C, hs-CRP, and creatinine, the risk for NAFLD increased across the homocysteine quartiles, and the OR in the highest quartile compared with the lowest quartile was 2.08 (95\% CI 1.61, 2.67). Similar results were also observed when homocysteine was considered as a continuous exposure variable (per SD increment, Table 2).

\section{Subgroup analyses}

To determine the effect of potential confounding factors, the associations between quartiles of homocysteine and NAFLD were further investigated among subgroups. As a result, we determined that the association differed significantly according to gender, BMI category and smoking status ( $P$ for interaction: $0.001,0.002$ and $<0.001$, respectively; Fig. 1). No effect modifications by age, hypertension, or diabetes on the association between homocysteine and NAFLD were observed (all $P$ for interaction $>0.05$ ).

The subgroup analysis by gender indicated that homocysteine was strongly associated with NAFLD in females but was weaker in males (Fig. 1). After multivariate adjustment, the OR of having NAFLD with per SD increment of $\log$ homocysteine was 1.67 (95\% CI 1.40, 1.99) in females, whereas the corresponding OR was 1.14 (95\% CI 1.02, 1.27) in males. Similarly, obesity and non-smoking status were also related to an enhanced association between homocysteine and the prevalence of NAFLD (Fig. 1).

\section{Discussion}

In this cross-sectional study, the main finding was that elevated homocysteine levels were positively associated with the prevalence of NAFLD in Chinese adults. Besides, in the subgroup analyses, an effect modification by gender, BMI and smoking on the association was found. A stronger association of homocysteine with the prevalence of NAFLD was observed in female, obese and non-smoking adults than in male, normal weight and smoking subjects. 
Table 1 Characteristics of the study population in total and according to homocysteine quartiles

\begin{tabular}{|c|c|c|c|c|c|c|}
\hline \multirow[t]{2}{*}{ Variables } & \multirow[t]{2}{*}{ Total } & \multicolumn{4}{|c|}{ Quartiles of Homocysteine } & \multirow[t]{2}{*}{$P$ value } \\
\hline & & Q1 $(<5.1)$ & Q2 (5.1 to <7.1) & Q3 (7.1 to <9.9) & Q4 ( $\geq 9.9)$ & \\
\hline$N$ & 7203 & 1876 & 1815 & 1697 & 1815 & \\
\hline Homocysteine $(\mu \mathrm{mol} / \mathrm{L})^{\mathrm{a}}$ & $7.0(5.0,9.9)$ & $4.0(3.9,5.0)$ & $6.0(5.9,7.0)^{\#}$ & $8.3(7.9,9.0)^{\#}$ & $12.3(10.8,14.9)^{\#}$ & $<0.001$ \\
\hline Age (years) ${ }^{a}$ & $49.0(40.0,59.0)$ & $46.0(38.0,52.0)$ & $49.0(40.0,57.0)^{\#}$ & $48.0(39.0,58.0)^{\#}$ & $53.0(42.0,70.0)^{\#}$ & $<0.001$ \\
\hline Male, \% & $3801(52.8)$ & $477(25.4)$ & $794(43.7)^{\#}$ & $1024(60.3)^{\#}$ & $1506(83.0)^{\#}$ & $<0.001$ \\
\hline $\mathrm{BMI}\left(\mathrm{kg} / \mathrm{m}^{2}\right)$ & $24.1 \pm 3.3$ & $23.6 \pm 3.2$ & $24.0 \pm 3.2^{\#}$ & $24.2 \pm 3.2^{\#}$ & $24.7 \pm 3.3^{\#}$ & $<0.001$ \\
\hline Current smoker, \% & $1698(24.0)$ & $284(15.5)$ & $364(20.6)^{\#}$ & $439(26.2)^{\#}$ & $611(33.9)^{\#}$ & $<0.001$ \\
\hline Physical activity & & & & & & $<0.001$ \\
\hline Inactive, \% & $3590(53.6)$ & $991(58.7)$ & $888(54.0)$ & $898(55.6)$ & $813(46.4)$ & \\
\hline Moderate, \% & $1769(26.4)$ & $461(27.3)$ & $472(28.7)$ & $408(25.2)$ & $428(24.4)$ & \\
\hline Active, $\%$ & $1342(20.0)$ & $235(13.9)$ & $284(17.3)$ & $310(19.2)$ & $513(29.2)$ & \\
\hline Education & & & & & & $<0.001$ \\
\hline Illiteracy/Primary, \% & $282(4.1)$ & $74(4.2)$ & $62(3.6)$ & $72(4.4)$ & $74(4.2)$ & \\
\hline Middle school, \% & $2234(32.5)$ & $683(38.3)$ & $612(35.8)$ & $479(29.4)$ & $460(26.2)$ & \\
\hline College or higher, \% & $4361(63.4)$ & $1026(57.5)$ & $1035(60.6)$ & $1081(66.2)$ & $1219(69.5)$ & \\
\hline Drinking, \% & $1459(20.3)$ & $252(13.4)$ & $276(15.2)$ & $412(24.3)^{\#}$ & $519(28.6)^{\#}$ & $<0.001$ \\
\hline Hypertension, \% & $2144(30.0)$ & $342(18.4)$ & $485(27.0)^{\#}$ & $507(30.1)^{\#}$ & $810(45.0)^{\#}$ & $<0.001$ \\
\hline Diabetes, $\%$ & $543(7.5)$ & $115(6.1)$ & $132(7.3)$ & $127(7.5)$ & $169(9.3)^{\#}$ & 0.002 \\
\hline $\operatorname{ALT}(U / L)^{a}$ & $21.0(15.0,30.0)$ & $18.0(14.0,26.0)$ & $20.0(15.0,29.0)^{\#}$ & $22.0(17.0,33.0)^{\#}$ & $23.0(17.0,33.0)^{\#}$ & $<0.001$ \\
\hline $\operatorname{TBIL}(\mu \mathrm{mol} / \mathrm{L})^{\mathrm{a}}$ & $14.8(12.1,18.3)$ & $14.1(11.6,17.1)$ & $14.7(11.9,18.2)^{\#}$ & $15.1(12.3,18.8)^{\#}$ & $15.6(12.5,19.1)^{\#}$ & $<0.001$ \\
\hline ALB $(g / L)$ & $46.5 \pm 2.6$ & $46.2 \pm 2.5$ & $46.6 \pm 2.5^{\#}$ & $46.8 \pm 2.6^{\#}$ & $46.5 \pm 2.8^{\#}$ & $<0.001$ \\
\hline $\operatorname{PLT}\left(10^{9} / \mathrm{L}\right)$ & $213.1 \pm 55.0$ & $218.2 \pm 55.0$ & $215.8 \pm 55.5$ & $215.5 \pm 56.7$ & $202.9 \pm 51.6^{\#}$ & $<0.001$ \\
\hline Uric acid $(\mu \mathrm{mol} / \mathrm{L})$ & $297.7 \pm 92.2$ & $263.8 \pm 84.5$ & $289.6 \pm 91.9^{\#}$ & $300.5 \pm 86.0^{\#}$ & $338.3 \pm 90.0^{\#}$ & $<0.001$ \\
\hline $\mathrm{TG}(\mathrm{mmol} / \mathrm{L})^{\mathrm{a}}$ & $1.3(0.9,1.9)$ & $1.2(0.8,1.7)$ & $1.3(0.9,1.8)^{\#}$ & $1.3(0.9,2.0)^{\#}$ & $1.4(1.0,2.0)^{\#}$ & $<0.001$ \\
\hline $\mathrm{TC}(\mathrm{mmol} / \mathrm{L})$ & $5.2 \pm 1.0$ & $5.1 \pm 1.0$ & $5.2 \pm 1.0^{\#}$ & $5.2 \pm 1.0^{\#}$ & $5.1 \pm 1.0$ & 0.001 \\
\hline $\mathrm{HDL}-\mathrm{C}(\mathrm{mmol} / \mathrm{L})$ & $1.6 \pm 0.4$ & $1.7 \pm 0.4$ & $1.6 \pm 0.4^{\#}$ & $1.6 \pm 0.4^{\#}$ & $1.5 \pm 0.4^{\#}$ & $<0.001$ \\
\hline $\mathrm{hs}-\mathrm{CRP}(\mathrm{mg} / \mathrm{L})^{\mathrm{a}}$ & $1.1(0.6,1.9)$ & $0.9(0.5,1.7)$ & $1.1(0.6,2.0)^{\#}$ & $1.1(0.6,2.0)^{\#}$ & $1.2(0.7,2.1)^{\#}$ & $<0.001$ \\
\hline Creatinine $(\mu \mathrm{mol} / \mathrm{L})$ & $67.2 \pm 22.0$ & $57.4 \pm 12.2$ & $64.6 \pm 18.9^{\#}$ & $67.9 \pm 15.0^{\#}$ & $79.4 \pm 30.7^{\#}$ & $<0.001$ \\
\hline Prevalence of NAFLD, \% & $2370(32.9)$ & $372(19.8)$ & $513(28.3)^{\#}$ & $640(37.7)^{\#}$ & $845(46.6)^{\#}$ & $<0.001$ \\
\hline
\end{tabular}

Abbreviations: $B M I$ body mass index, $A L T$ alanine transaminase, $T B I L$ total bilirubin, $A L B$ albumin, $P L T$ platelet count, $T G$ triglyceride, $T C$ total cholesterol, $H D L-C$ highdensity lipoprotein cholesterol, $h s$-CRP high-sensitivity C-reactive protein

${ }^{a}$ Variables were log transformed before analysis

${ }^{\#} P<0.05$, in comparison with the reference group (quartile 1). $P$ values were corrected by Bonferroni's method due to multiple testing

Over the past decade, accelerating interest has developed in the relationship between homocysteine and the prevalence of NAFLD. On one hand, homocysteine is a sulfhydryl-containing amino acid mainly produced and catabolized in the liver $[8,9]$. It is plausible that, in the presence of liver damage, alterations of serum homocysteine levels may occur. On the other hand, elevated homocysteine may conversely promote the progression of liver damage. Indeed, Yao et al. [23] recently found that hyperhomocysteinemia could promote hepatic steatosis in mice through activation of the aryl hydrocarbon receptor/CD36 pathway. According to several experimental studies, hepatic lipid accumulation was induced in different models of hyperhomocysteinemia [24-26].
In a study of pediatric NAFLD, Pastore et al. [27] found that homocysteine strongly correlated with the severity of liver damage. These previous studies suggested that homocysteine was associated with NAFLD progression. Meanwhile, accumulating evidence has demonstrated that homocysteine is an independent risk factor for cardiovascular diseases such as stroke $[28,29]$ and ischemic heart disease $[30,31]$, which suggests that homocysteine may mediate the association between NAFLD and cardiovascular diseases [32]. Thus, it is plausible that homocysteine might be an effective target for preventing NAFLD progression and its related cardiovascular complications.

Although several clinical studies have reported the association between homocysteine and the prevalence of 
Table 2 Odds ratios for the association between serum homocysteine and the prevalence of non-alcoholic fatty liver disease

\begin{tabular}{|c|c|c|c|c|}
\hline \multirow{2}{*}{$\begin{array}{l}\text { Homocysteine } \\
(\mu \mathrm{mol} / \mathrm{L})\end{array}$} & \multirow[t]{2}{*}{$N=7023$} & \multicolumn{3}{|l|}{ OR $(95 \% \mathrm{Cl})$} \\
\hline & & Model 1 & Model 2 & Model 3 \\
\hline \multicolumn{5}{|l|}{ Quartiles } \\
\hline Q1 $(<5.1)$ & 1876 & 1 (Ref) & 1 (Ref) & 1 (Ref) \\
\hline Q2 $(5.1$ to $<7.1)$ & 1815 & $1.52(1.30,1.77)$ & $1.44(1.18,1.74)$ & $1.32(1.04,1.67)$ \\
\hline Q3 (7.1 to <9.9) & 1697 & $2.38(2.04,2.76)$ & $1.90(1.57,2.30)$ & $1.75(1.39,2.22)$ \\
\hline Q4 ( $\geq 9.9)$ & 1815 & $3.10(2.67,3.59)$ & $1.91(1.57,2.32)$ & $2.08(1.61,2.67)$ \\
\hline$P$ for trend & & $<0.001$ & $<0.001$ & $<0.001$ \\
\hline Per SD increment & 7023 & $1.47(1.40,1.55)$ & $1.18(1.10,1.27)$ & $1.25(1.15,1.37)$ \\
\hline$P$ value & & $<0.001$ & $<0.001$ & $<0.001$ \\
\hline
\end{tabular}

Model 1: adjusted for age ${ }^{\mathrm{a}}$; Model 2: further adjusted for gender, body mass index, current smoker, physical activity, education, drinking; Model 3: further adjusted for hypertension, diabetes, uric acid, alanine transaminase ${ }^{\mathrm{a}}$, total bilirubin ${ }^{\mathrm{a}}$, albumin, platelet count, triglyceride ${ }^{\mathrm{a}}$, total cholesterol, high-density lipoprotein cholesterol, high-sensitivity C-reactive protein ${ }^{\mathrm{a}}$, creatinine.

Per SD increment represents 1 SD increment of log homocysteine.

aariables were log transformed before analysis

NAFLD, the results were inconclusive. According to a case-control study including 50 patients with nonalcoholic steatohepatitis (NASH) and 30 healthy subjects, serum homocysteine levels were identified significantly higher in patients with NASH vs. controls [15]. Won et al. [33] found that the uppermost quartile of homocysteine in men was significantly associated with a 6.78 -fold increased OR for NAFLD. Consistent with these findings, the positive association of serum homocysteine levels with NAFLD was shown in a meta-analysis by Dai et al. [34], which was based on eight studies (six cross- sectional and two case-control), totaling 935 participants (397 NAFLD cases and 538 controls). In contrast, another study suggested that serum homocysteine levels were not associated with NAFLD [35]. Furthermore, most related studies did not assess the independent risk of homocysteine with adjusting for potential confounders such as BMI, liver enzymes and dyslipidemia, and the relatively small number of subjects in these studies also made it difficult to draw definitive conclusions. The results of our study using a large sample with adjustment for multiple confounders support a

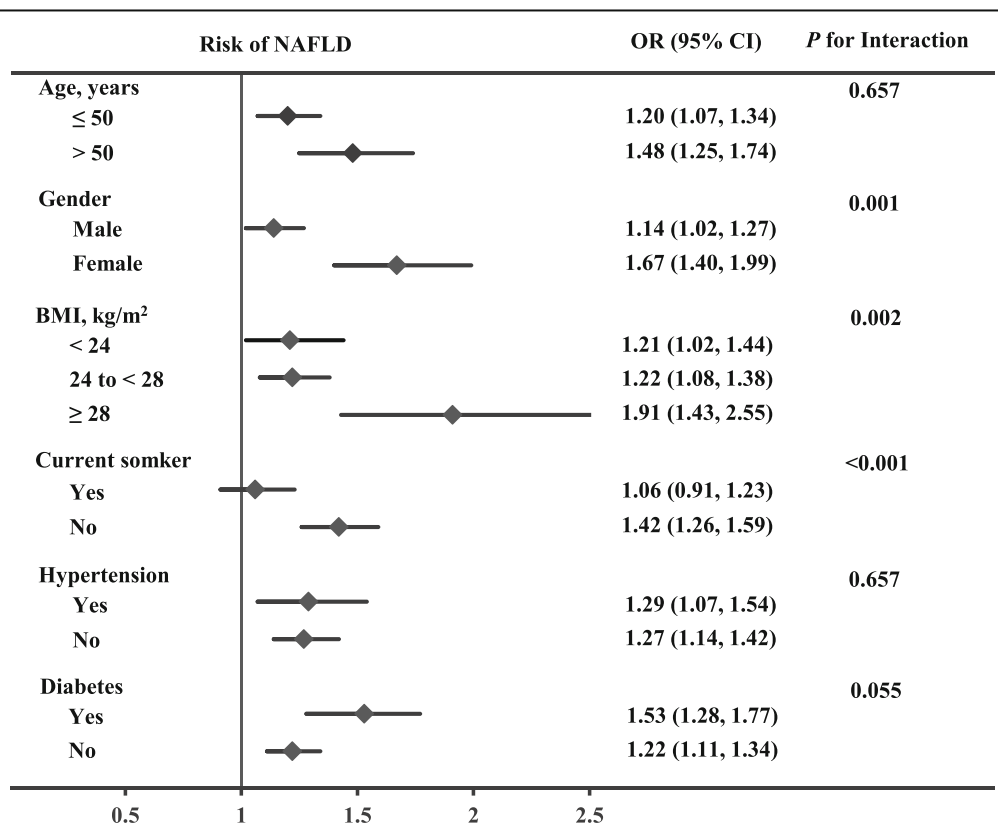

Fig. 1 Association between homocysteine and non-alcoholic fatty liver disease in selected subgroups. Homocysteine is presented with a continuous scale (per SD increment of log homocysteine). Multiple logistic regression and interaction adjusted for age ${ }^{\mathrm{a}}$, gender, body mass index, current smoker, physical activity, education, drinking, hypertension, diabetes, uric acid, alanine transaminase ${ }^{a}$, total bilirubina ${ }^{a}$ albumin, platelet count, triglyceride ${ }^{a}$, total cholesterol, high-density lipoprotein cholesterol, high-sensitivity C-reactive protein ${ }^{a}$, and creatinine. ${ }^{2}$ Variables were log transformed before analysis 
positive association between homocysteine and the prevalence of NAFLD.

Our study also explored a possible effect modification by other related factors, such as gender and BMI, on the association between homocysteine and the prevalence of NAFLD. We concluded that the association was stronger in female, obese or non-smoking adults. In contrast to our study, a recent study found that homocysteine was closely associated with the prevalence of NAFLD in men but not in women [33]. However, only approximately 30 subjects with a small number of events were categorized in each quartile of homocysteine in that study, and it was hard to conduct a stable estimate for NAFLD. Interestingly, several previous studies have also suggested that serum homocysteine-related risk of cardiovascular diseases prone to be significant in women, but not in men [36-38]. Unfortunately, to date, the underlying mechanism of such gender-specific differences remains unknown. Obesity was reported to be associated with two key liver enzymes involved in homocysteine metabolism and may affect the catabolism of homocysteine in liver damage [39]. Our study firstly showed that the association between homocysteine and NAFLD was strengthened by obesity. Moreover, our study found that the association between homocysteine and NAFLD was attenuated in current smokers. Cigarette smoking is known to be associated with oxidative stress, the proinflammatory response, and fatty acid synthesis, which may have caused the phenomenon observed in our study $[40,41]$. However, further studies are required to validate and explicate the effect modification noted in our study.

The present study has several limitations. First, this cross-sectional study was unable to explain the causal relationship; thus, further prospective cohort studies and intervention trials should be undertaken to establish a causal association between homocysteine and the prevalence of NAFLD. Second, the presence of hepatic steatosis was assessed by ultrasonography rather than liver biopsy pathology. Nonetheless, ultrasonography has been widely accepted for population-based studies due to its safety, economical cost and reasonable accuracy [21, 42].

\section{Conclusions}

In conclusion, we found that homocysteine was significantly associated with the prevalence of NAFLD, particularly in female, obese or non-smoking adults. Further research is needed to determine whether the associations found in this study are causal and whether homocysteinelowering therapy can be used to prevent progressive NAFLD and its related cardiovascular complications, particularly for female, obese and non-smoking adults.

\section{Abbreviations}

ALT: Alanine transaminase; BMI: Body mass index; BP: Blood pressure

$\mathrm{Cl}$ : Confidence interval; HDL-C: High-density lipoprotein cholesterol; hs-
CRP: High-sensitivity C-reactive protein; NAFLD: Non-alcoholic fatty liver disease; OR: Odds ratio; TBIL: Total bilirubin; TC: Total cholesterol; TG: Triglyceride

\section{Acknowledgments}

We are grateful to thank the Center of Health Management for their help with volunteer recruitment and measurement. We also wish to thank all of the study participants for their cooperation and participation.

\section{Funding}

This research was supported by the National Science and Technology Major Projects for "Major New Drugs Innovation and Development" (2012ZX09303014001), the Planned Science and Technology Project of Hunan Province, China (2014 weeks), and the Fundamental Research Funds for the Central Universities of Central South University (2016zzts153).

\section{Availability of data and materials}

The datasets during the current study available from the corresponding author on reasonable request.

\section{Authors' contribution}

Conception and design: HY, YL, and HD. Acquisition of the data: ZC. Data analysis and interpretation of the data: HD and WW. Drafting the manuscript or revising it critically for important intellectual content: HD, XT, and RC. All authors read and approved the final manuscript.

\section{Competing interests}

The authors declare that they have no competing interests.

\section{Consent for publication}

Not applicable.

\section{Ethics approval and consent to participate}

This study was approved by the Medical Ethics Committee of Third Xiangya Hospital, and each participant provided written informed consent before participating. All of the protocols and procedures were performed according to the Declaration of Helsinki.

\section{Author details}

${ }^{1}$ Center of Clinical Pharmacology, the Third Xiangya Hospital, Central South University, 138 Tong-Zi-Po Road, Changsha, Hunan Zip 410013, People's Republic of China. ${ }^{2}$ Center of Health Management, the Third Xiangya Hospital, Central South University, Changsha, Hunan Province Zip 410013, People's Republic of China. ${ }^{3}$ Division of Gastroenterology, Union Hospital, Huazhong University of Science and Technology, Wuhan, Hubei Province Zip 430022, People's Republic of China.

Received: 24 August 2016 Accepted: 25 November 2016

Published online: 12 December 2016

\section{References}

1. Younossi ZM, Koenig AB, Abdelatif D, Fazel Y, Henry L, Wymer M. Global epidemiology of nonalcoholic fatty liver disease-Meta-analytic assessment of prevalence, incidence, and outcomes. Hepatology. 2016;64:73-84.

2. Fan JG. Epidemiology of alcoholic and nonalcoholic fatty liver disease in China. J Gastroenterol Hepatol. 2013;28 Suppl 1:11-7.

3. Li Z, Xue J, Chen P, Chen L, Yan S, Liu L. Prevalence of nonalcoholic fatty liver disease in mainland of China: a meta-analysis of published studies. J Gastroenterol Hepatol. 2014;29:42-51.

4. Wong W, Chu WC, Wong GL, Chan RS, Chim AM, Ong A, et al. Prevalence of non-alcoholic fatty liver disease and advanced fibrosis in Hong Kong Chinese: a population study using proton-magnetic resonance spectroscopy and transient elastography. Gut. 2012;61:409-15.

5. Musso G, Gambino R, Tabibian JH, Ekstedt M, Kechagias S, Hamaguchi M, et al. Association of non-alcoholic fatty liver disease with chronic kidney disease: a systematic review and meta-analysis. PLoS Med. 2014;1 1:e1001680.

6. Targher G, Byrne CD, Lonardo A, Zoppini G, Barbui C. Non-alcoholic fatty liver disease and risk of incident cardiovascular disease: a meta-analysis. J Hepatol. 2016;65:589-600. 
7. Mikolasevic I, Racki S, Zaputovic L, Lukenda V, Sladoje-Martinovic B, Orlic L. Nonalcoholic fatty liver disease (NAFLD) and cardiovascular risk in renal transplant recipients. Kidney Blood Press Res. 2014;39:308-14.

8. Wijekoon EP, Brosnan ME, Brosnan JT. Homocysteine metabolism in diabetes. Biochem Soc Trans. 2007;35:1175-9.

9. Williams KT, Schalinske KL. Homocysteine metabolism and its relation to health and disease. Biofactors. 2010;36:19-24.

10. Ganguly P, Alam SF. Role of homocysteine in the development of cardiovascular disease. Nutr J. 2015;14:6.

11. Werstuck GH, Lentz SR, Dayal S, Hossain GS, Sood SK, Shi YY, et al. Homocysteineinduced endoplasmic reticulum stress causes dysregulation of the cholestero and triglyceride biosynthetic pathways. J Clin Invest. 2001;107:1263-73.

12. Obeid R, Herrmann W. Homocysteine and lipids: S-adenosyl methionine as a key intermediate. FEBS Lett. 2009;583:1215-25.

13. Hirsch S, Poniachick J, Avendano M, Csendes A, Burdiles P, Smok G, et al. Serum folate and homocysteine levels in obese females with non-alcoholic fatty liver. Nutrition. 2005;21:137-41.

14. de Carvalho SC, Muniz MT, Siqueira MD, Siqueira ER, Gomes AV, Silva KA, et al. Plasmatic higher levels of homocysteine in non-alcoholic fatty liver disease (NAFLD). Nutr J. 2013;12:37.

15. Leach NV, Dronca E, Vesa SC, Sampelean DP, Craciun EC, Lupsor M, et al. Serum homocysteine levels, oxidative stress and cardiovascular risk in non-alcoholic steatohepatitis. Eur J Intern Med. 2014;25:762-7.

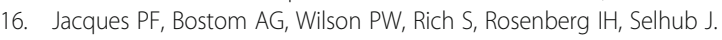
Determinants of plasma total homocysteine concentration in the Framingham Offspring cohort. Am J Clin Nutr. 2001;73:613-21.

17. Farrell GC, Chitturi S, Lau GK, Sollano JD, Asia-Pacific Working Party on N. Guidelines for the assessment and management of non-alcoholic fatty liver disease in the Asia-Pacific region: executive summary. J Gastroenterol Hepatol. 2007;22:775-7.

18. Dai H, Lu S, Tang X, Lu M, Chen R, Chen Z, et al. Combined association of serum uric acid and metabolic syndrome with chronic kidney disease in hypertensive patients. Kidney Blood Press Res. 2016;41:413-23.

19. Wei J, Lei GH, Fu L, Zeng C, Yang T, Peng SF. Association between dietary Vitamin C intake and non-alcoholic fatty liver disease: a crosssectional study among middle-aged and older adults. PLoS One. 2016; 11:e0147985.

20. Fan JG, Jia JD, Li YM, Wang BY, Lu LG, Shi JP, et al. Guidelines for the diagnosis and management of nonalcoholic fatty liver disease: update 2010. J Dig Dis. 2011;12:38-44. published in Chinese on Chinese Journal of Hepatology 2010; 18:163-166.

21. Chalasani N, Younossi Z, Lavine JE, Diehl AM, Brunt EM, Cusi K, et al. The diagnosis and management of non-alcoholic fatty liver disease: practice guideline by the American association for the study of liver diseases, American college of gastroenterology, and the American gastroenterological association. Hepatology. 2012;55:2005-23.

22. Zhou BF, Cooperative Meta-Analysis Group of the Working Group on Obesity in C. Predictive values of body mass index and waist circumference for risk factors of certain related diseases in Chinese adults-study on optimal cut-off points of body mass index and waist circumference in Chinese adults. Biomed Environ Sci. 2002;15:83-96.

23. Yao L, Wang C, Zhang X, Peng L, Liu W, Zhang X, et al. Hyperhomocysteinemia activates the aryl hydrocarbon receptor/CD36 pathway to promote hepatic steatosis in mice. Hepatology. 2016;64:92-105.

24. Woo CW, Siow YL, Pierce GN, Choy PC, Minuk GY, Mymin D, et al, Hyperhomocysteinemia induces hepatic cholesterol biosynthesis and lipid accumulation via activation of transcription factors. Am J Physiol Endocrinol Metab. 2005;288:E1002-10

25. Namekata K, Enokido Y, Ishii I, Nagai Y, Harada T, Kimura H. Abnormal lipid metabolism in cystathionine beta-synthase-deficient mice, an animal model for hyperhomocysteinemia. J Biol Chem. 2004;279:52961-9.

26. Hamelet J, Demuth K, Paul JL, Delabar JM, Janel N. Hyperhomocysteinemia due to cystathionine beta synthase deficiency induces dysregulation of genes involved in hepatic lipid homeostasis in mice. J Hepatol. 2007:46:151-9.

27. Pastore A, Alisi A, di Giovamberardino G, Crudele A, Ceccarelli S, Panera N, et al. Plasma levels of homocysteine and cysteine increased in pediatric NAFLD and strongly correlated with severity of liver damage. Int J Mol Sci. 2014;15:21202-14.

28. Zhang W, Sun K, Chen J, Liao Y, Qin Q, Ma A, et al. High plasma homocysteine levels contribute to the risk of stroke recurrence and all-cause mortality in a large prospective stroke population. Clin Sci. 2010;118:187-94.
29. He Y, Li Y, Chen Y, Feng L, Nie Z. Homocysteine level and risk of different stroke types: a meta-analysis of prospective observational studies. Nutr Metab Cardiovasc Dis. 2014;24:1158-65.

30. Homocysteine Studies C. Homocysteine and risk of ischemic heart disease and stroke: a meta-analysis. JAMA. 2002;288:2015-22.

31. Humphrey LL, Fu R, Rogers K, Freeman M, Helfand M. Homocysteine level and coronary heart disease incidence: a systematic review and metaanalysis. Mayo Clin Proc. 2008:83:1203-12.

32. Francque SM, van der Graaff D, Kwanten WJ. Non-alcoholic fatty liver disease and cardiovascular risk: Pathophysiological mechanisms and implications. J Hepatol. 2016:65:425-43.

33. Won BY, Park KC, Lee SH, Yun SH, Kim MJ, Park KS, et al. Sex difference in the association between serum homocysteine level and non-alcoholic fatty liver disease. Korean J Fam Med. 2016;37:242-7.

34. Dai Y, Zhu J, Meng D, Yu C, Li Y. Association of homocysteine level with biopsy-proven non-alcoholic fatty liver disease: a meta-analysis. J Clin Biochem Nutr. 2016;58:76-83.

35. Polyzos SA, Kountouras J, Anastasilakis AD, Margouta A, Mantzoros CS. Association between circulating irisin and homocysteine in patients with nonalcoholic fatty liver disease. Endocrine. 2015;49:560-2.

36. Kuo HK, Yen CJ, Bean JF. Levels of homocysteine are inversely associated with cardiovascular fitness in women, but not in men: data from the national health and nutrition examination survey 1999-2002. J Intern Med. 2005:258:328-35.

37. Wang C, Han L, Wu Q, Zhuo R, Liu K, Zhao J, et al. Association between homocysteine and incidence of ischemic stroke in subjects with essential hypertension: a matched case-control study. Clin Exp Hypertens. 2015;37:557-62.

38. Zhong C, Xu T, Xu T, Peng Y, Wang A, Wang J, et al. Plasma homocysteine and prognosis of acute ischemic stroke: a gender-specific analysis from CATIS randomized clinical trial. Mol Neurobiol 2016. doi: 10.1007/s12035-016-9799-0.

39. Fonseca V, Dicker-Brown A, Ranganathan S, Song W, Barnard RJ, Fink L, et al. Effects of a high-fat-sucrose diet on enzymes in homocysteine metabolism in the rat. Metabolism. 2000:49:736-41.

40. Zein CO, Unalp A, Colvin R, Liu YC, McCullough AJ, Nonalcoholic Steatohepatitis Clinical Research Network. Smoking and severity of hepatic fibrosis in nonalcoholic fatty liver disease. J Hepatol. 2011;54:753-9.

41. Chen S, Wu P, Zhou L, Shen Y, Li Y, Song H. Relationship between increase of serum homocysteine caused by smoking and oxidative damage in elderly patients with cardiovascular disease. Int J Clin Exp Med. 2015;8:4446-54.

42. Xu C, Yu C, Ma H, Xu L, Miao M, Li Y. Prevalence and risk factors for the development of nonalcoholic fatty liver disease in a nonobese Chinese population: the Zhejiang Zhenhai Study. Am J Gastroenterol. 2013;108: 1299-304.

\section{Submit your next manuscript to BioMed Central and we will help you at every step:}

- We accept pre-submission inquiries

- Our selector tool helps you to find the most relevant journal

- We provide round the clock customer support

- Convenient online submission

- Thorough peer review

- Inclusion in PubMed and all major indexing services

- Maximum visibility for your research

Submit your manuscript at www.biomedcentral.com/submit
) Biomed Central 